\title{
DAS ADJEKTIV IN „SYR GAWAYN AND THE GRENE KNYZT".
}

(Fortsetzung.)

\section{Das adjektiv im satze (syntax).}

Von der betrachtung des adjektivs als einzelwortes in der formenlehre und der gruppen aus adj. plus sinnesobjekt in der stilistik schreitet die darstellung fort zur nächsthöhern einheit, zum adj. in satze.

Es sei hier an das in der einleitung gesagte erinnert, wonach dieser dritte hauptteil der arbeit in sechs abschnitte zerfallen und der reihe nach behandeln soll: das attributive, appositive, prädikative und substantivierte adj., zusätze und ergänzungen und endlich nachsätze beim adj.

Zur weitern gliederung vergleiche man ebendort.

\section{Das attributive adjektiv.}

$\$ 35 . \quad$ 1. Art und zahl der verwendeten adjektiva und bemerkenswertes im gebrauche.

(jeht man alle die in der materialsammlung zusammengetragenen fälle einmal durch, in denen sich ein adj. in attributiver stellung bei einem substantiv findet, so kann man nicht verfehlen zu bemerken, dafs die verschiedenen, an der bildung des adjektivschatzes beteiligten sprachen ganz verschieden stark vertreten sind, u. z. auch unabhängig von dem malse, in dem sie einzeln zu diesem wortschatze beitragen. Ferner wird einem auffallen, dafs die in der stilistik abgeleiteten sechs adjektivgruppen ebenfalls in ganz ungleicher weise, wiederum nicht einfach proportional der grölse der 
gruppe, an der attribuierung beteiligt sind. Eine genaue prüfung dieser fragen fübrt nun zu folgenden ergebnissen:

In attributiver verwendung kommen altenglische adjektiva in 509 fällen, altfrz. in 108, altnord. in 35, sonstige germ. in 11 , keltische in 2 , abgeleitete in 61 und zusammengesetzte adjektiva in 6 fällen vor: Summa 732 .

Die sechs adjektivgruppen sind an der attribuierung wie folgt beteiligt:

1. sinnenfällige eigenschaften $170 \mathrm{mal}, 2$. wesenseigenschaften $227 \mathrm{mal}$, 3. zustandsangaben $43 \mathrm{mal}$, 4. verhältnisbestimmungen $83 \mathrm{mal}, 5$. ästhetische bestimmungen $131 \mathrm{mal}$, 6. sonstiger gefühlsanteil $76 \mathrm{mal}$ : Summa 730 .

Es ist von interesse zu untersuchen, in welchem malse attribuierung bei substantiven, die lebewesen bezeichnen, also zu allermeist der personen des stückes, stattfindet und in welchem verhältnis sie zur gesamten attribuierung steht. Das anfänglich etwas ïberraschende ergebnis ist $186: 730$, also ganz wenig mehr als ein viertel. Doch muls man das bedenken, worauf schon Scheinert in seiner einleitung ganz richtig hinweist: "Bei sächlichen konkreten mufs natürlich der beschreibende, direkte stil grölsern raum einnehmen; lebewesen können und müssen indirekt vorgeführt werden und sich charakterisieren." Es ist sehr zu bedauern, daIs Scheinert sich auf v. $1-1250$, also auf $5 / 12$ des ganzen Beowulf, bei seiner untersuchung der syntaktischen verhältnisse beschränkt hat. Dadurch ist es mir unmöglich gemacht, hier und in folgenden lehrreiche vergleiche mit Beowulf anzustellen. Noch weniger könnte ich andre werke heranziehen, da mir keine einzelstudie bekannt geworden, die entsprechendes material so vollständig böte, wie hier versucht werden soll; vielmehr bleiben sie alle noch hinter Scheinert zurück.

Eine anzahl attributivisch gebrauchter adjektiva scheinen besondrer hervorhebung wert, sei es nun auf grund eines funktions- oder eines bedentungswandels, den sie im laufe der sprachentwicklung durchgemacht haben. Zur ersten art gehören "sere" und als substantiva und adjektiva verwendbare wörter wie chef, daynté, outrage, prynce, wynne, für die ich auf den schlufs der materialsammlung (§ 18) zurückverweise.

Das adjektivisch und adverbiell gebrauchte "sere" aus dem Altnord. ist ursprünglich weder das eine noch das andre, 
DAS ADJEKTIV IN "SYR GAWAYN AND THE GRENE KNYZT". 16:)

sondern der in analogie zur ersten und zweiten person des persönlichen fürwortes gebildete dativ des rückbezüglichen fürwortes und wird in der bedeutung "several, diverse" gebraucht. Dabei ist beachtenswert, dals es verbunden werden kann nicht nur mit substantiven im plural, sondern auch im sing.: His sere pyne 1985. So verwendet noch Milt on manchmal ein singularisches subst. bei "several": His s. way (Par. lost II).

Auch zu "mid-" bietet er eine parallele in "mean", das er in dem häufig vorkommenden "mean while" wohl sicher noch attributivisch empfand.

Die wichtigsten fälle von bedeutungswandel liegen vor bei "much, littel, foo". Die von Einenkel für Chaucer belegten "more, most. less, lest" im sinne von grölser und kleiner finden sich in gleicher bedeutung im $S . G$. Die klarsten fälle sind: In de more half of his schelde 649 , prayse at more prys 1850, most renoun 231; "lasse" ist zufällig nur appositiv und prädikativ belegt, z. b. hit (bytte) watz no lasse 2226 , de lest lachet ou[d]er loupe 591. Den für Chaucer in der bedeutung "grofs" nicht angefïhrten positiv "much" haben wir im $S$. G. in den beispielen: a much berd as a busk 182, denn he melez muryly wyð a much steuen 2336. "Foo", ae. fah, kommt 1430 in der bedeutung "rough" vor: a foo cragge, daneben aber, 816, substantiviert in dem üblichen sinne von "feind". Angaben über ausschliefslichen oder überwiegenden gebranch gewisser wörter in attributiver oder prädikativer stellung, wie sie sich bei Wendt finden, der die gesamte lebende sprache berïcksichtigt, kann natürlich eine einzelstudie nicht zu machen wagen. "Goode" = ziemlich, passend, wie Einenkel es für Chaucer aufführt, ist in unserm texte so nicht zu belegen; auf personen angewendet bedeutet es wacker, trefflich, redlich, auch lieb und wert, und bei sachen heifst es gut. Zu "veray" vergleiche man unter "adj. plus adv." (\$ 50).

\section{$\S 36$. 2. Stellung des attributiven adjektivs.}

Bei der frage nach der stellung unterscheidet man zweckmä Ssig zwei hauptfälle: Das subst. ist 1 . von einem, oder 2 . von zwei (ganz selten mehr) adjektiven begleitet. Für 1. bestehen die zwei möglichkeiten, dals es vor oder nach dem subst. steht, während 2. die drei möglichkeiten zuläIst: Beide 
adjektiva stehen vor oder beide nach, oder sie gruppieren sich um ihr beziehungswort. Schliefslich können die adjektiva verbunden oder unverbunden neben einander stehen. Folgendes ist nun das ergebnis einer auf all diese möglichkeiten achtenden untersuchung für den $S . G$. (A= adjektiv, $\mathrm{S}=$ substantiv $)$ :

$\mathrm{A}+\mathrm{S} 581 \mathrm{mal}, \mathrm{S}+\mathrm{A} 116 ; 2 \mathrm{~A}+\mathrm{S} 16, \mathrm{~S}+2 \mathrm{~A} 4$; $\mathrm{A}+\mathrm{S}+\mathrm{A} 13$ mal. Summa: 730 .

Beispiele für den hauptfall 2, der ihrer allein bedürftig scheint, sind: 2 a: Bryzt brode scheldez, verbindung durch "and" kommt nicht vor. $2 \mathrm{~b}$ : Destines derf and dere 564; ganz analog, also verbunden, auch die drei übrigen stellen. $2 \mathrm{c}$ : Hyze bonkkez and brent $2165 ; 8$ von den 13 vorhandenen fällen weisen "and" auf. A grene hors gret and dikke (175) ist mit bestimmtheit $\mathrm{zu}$ keinem der drei letztbesprochenen fälle $\mathrm{zu}$ stellen. Die weitern beispiele bringt der metrische teil, der auf die frage nach der stellung eines adjektivs hinter seinem beziehungswort eingehen wird. Hier genüge es festzustellen, dafs von den 116 fällen mit nachstehendem adj. sich 75 erklären lassen aus metrischen ursachen, deren wirkung zwar mehrfach durch zum adj. hinzutretende adverbien oder partikeln verstärkt wird. Auch das letzte viertel ist zur hälfte mit ziemlicher wahrscheinlichkeit als rhythmisch bedingt $\mathrm{zu}$ erweisen, sodafs als regelmälsige unbeeinflufste stellung des adjektivs durchaus die vor dem subst. zu gelten hat.

\section{§ 37. 3. Gebrauch des artikels beim attributiven adj.}

Dieser abschnitt zerfällt naturgemäls in die behandlung des bestimmten und unbestimmten artikels und soll in jedem der teile $\mathrm{zu}$ antworten suchen auf die frage: Wann steht der artikel beim attributiven adj., wann fehlt er? Nach der geschichtlichen entwicklung ist bei dem aus dem pronomen demonstrativ entstandenen bestimmten artikel eine dieser wortart nahe gebliebene gebrauchsart, eben ein schwacher hinweis, zu unterscheiden von einer später mehr und mehr zunehmenden verwendungsweise, nach der er als aussondernder, individualisierender artikel bezeichnet werden kann. Ebenfalls geschichtlichen gesichtspunkten entstammt beim unbestimmten. aus dem zahlwort ân entstandenen artikel die artbestimmung: 
zählender, einführender und absoluter oder allgemeiner unbestimmter artikel. Beispiele für alle arten aus dem $S$. G. sind:

Hinweisender bestimmter artikel : Je leue lorde of Je londe 1133, gret is đe gode gle mit konsekutivem "Jat" 1536, ठe leuest Jing mit relativischem "Jat" 1802.

Aussondernder bestimmter artikel: đe fre lorde 1156 (der grüne ritter nämlich), de fayre best 1359 (das vorher aus der zahl der andern schon ausgesondert).

Zählender unbestimmter artikel: ho razt hym a riche rynk of red golde werkez, wyd a starande ston ... 1817, 1818.

Einführender unbestimmter artikel: a littel dich 1709, (c wale tryster 1812 (neue data folgen im verlaufe der beschreibung).

Absoluter unbestimmter artikel: I were a knyzt kowarde 2131 ("knyzt kowarde" als allgemeiner begrift).

Da die fünf aufgeführten arten des artikels so vielfältige übergänge aufweisen, dals die zuweisung eines gegebenen falles an die eine oder andre schwierig und oft gar nicht durchführbar ist, so wird diese unterscheidung im folgenden nur soweit festgehalten werden, als sie das verständnis fördern kann.

a) Der bestimmte artikel.

c) Der artikel steht. Ich schliefse mich hier an A. Lichtenheld an, der in seiner arbeit "Das schwache adj. im Ags." (Zeitschr. für d. A. B. 16) hinsichtlich der funktion des bestimmten artikels zu folgendem ergebnis gekommen ist: "1. Er steht vor einem subst., zu dessen näherer bestimmung ein relativ- oder sonstiger erklärender beisatz folgt." Die besondern wendungen unter 2 . können hier übergangen werden. "3. Seine bei weitem häufigste funktion ist diese: Er deutet durch seinen hinzutritt an, dals ein subst. oder ebenso oft der begriff, den es enthält, im verlauf der erzählung schon genannt ist; hierher gehört auch der hinweis auf personen oder sachen, zu denen das durch das subst. ausgedrückte in engster beziehung steht. 4. Schon im Beourulf sind fälle, wo die beziehung des artikels im gedichte selbst fehlt. Diese weite demonstration kommt fast ausschliefslich bei gegenständen vor, die infolge ihrer rolle in sitte und leben für die damalige 
anschauung von besonderm interesse waren." Dieser 4., bezw. 3. fall Lichtenhelds erfährt für den $S$. $G$. einen ziemlich bedeutenden zuwachs durch die belege, in denen vom demonstrativen charakter des artikels noch weniger, oft gar nicht mehr, zu sprechen ist und wir den individualisierenden, blolsen bestimmten artikel haben. Nach Barnouw ist am zunehmenden gebrauche des artikels das abnehmende gefühl für die emphatische kraft des schwachen adjektivs so beteiligt: die verbindung art. + schw. adj. + subst. steigt proportional der abnahme der häufigkeit der verbindung art. + schw. adj. Natürlich kann beim flexionsverfall des $S$. G. vom schwachen adj. mit dem art. nicht gehandelt werden. Ich wende mich der erläuterung der drei aufgestellten fälle $\mathrm{zu}$.

1. Der best. art. hat hinweisende kraft und ist durch einen zu- oder nachsatz mitbedingt. Mit genitivischem zusatze: $e$ leue lorde of Je londe 1133, de lel layk of luf 1513, mony watz ðe myry moude of men and of houndez 1447, de hede of an elnzerde Je large lenkde hade 210. Mit einem nachsatze: Schewez hym Je schyre grece schorne vpon rybbes 1378 , gret is Je god gle ... Jat (konsekutiv 1536). In gleicher weise steht bei gesteigertem adj. der art., auch dann häufig, wenn kein solcher zu- oder nachsatz folgt, der aber, auch unausgesprochen, im zusammenhang des vergleiches doch wirklich vorliegt. Wherfore de better burne me burde be called 2278, 才e best burne ay abof 73, бe first cors 116; aber mit nachsatz z. b.: Je fiyrst word Jat he warp 224, ähnlich 2373. And Je loue-lokkest ladies Jat euev lif haden 52, ähnlich 53, 51; On de fautlest freke Jat euer on fote zede 2363, aber eine parallelstelle 1439 ohne nachsatz. $I$ wolde $I$ hade here Je leuest Jing for $\delta y$ luf Jat $I$ in londe welde 1801-2. And sayde he wat je velcomest wyze of de worlde 938, hit watz de myviest mute dat euer men herde 1915. Es zeigt sich also, dafs das attributiv gebrauchte adj. mit bestimmtem art. bei einem subjektiv, objektiv und prädikativ verwendeten subst. auftritt.

2. Der art. ist gleichzeitig hin- und zurückweisender natur in einer anzahl von fällen, in denen er durch sein hinzutreten ein subst. als etwas aus dem zusammenhange der erzählung wohlbekanntes oder zu bekanntem in naher beziehung stehend bezeichnet. Je fre lorde 1156 (jedem leser ist es klar, dafs nur der schloisherr, der grüne ritter, gemeint sein kann). 
Ganz so verhält es sich bei: đe gay lady 1248, đe myry mon (Gawayn) 1263, ferner 1955, 1179, 2524, de lady luflych 1757. Je stif kyng his-seluen 107 . Wie bei den für die handlung wichtigen personen, gilt für das setzen des bestimmten artikels ein gleiches bei sonstigen hervortretenden, das interesse auf sich ziehenden lebewesen oder gegenständen der erzählung. ¿e fayre best 1359 (das eben besprochene), đe wylde swyn 1628, ðenne hondeled ðay ðe hoge hed 1633 (nämlich des wildschweins), ðe grene chapel 451, 454. ... むe comly castel 1366, むe brode zatez 2069 (natürlich des schlosses), ähnliche stellen 1432. 2145, 169.

Zur demonstrativen kraft des artikels kommt, wie diese fälle zeigen, hier die identifizierende und individualisierende, wie schon für einen teil der beispiele unter 1 hinzu.

Das hinweisende element tritt gegenüber dem aussondernden noch mehr zurück, wir nähern uns noch mehr dem durch häufigen gebrauch abgeschliffenen blofsen art. in fällen wie den folgenden, die nicht bestimmte, bekannte personen oder dinge der erzählung, sondern angaben ziemlich unbestimmt gelassener und oft ganz allgemeiner natur bringen: ðe hyљe kyng = himmelskönig (der sinn ist klar auch ohne vorherige erwähnung); đe breme bukkez 1155, de quyte snaw lay bisyde 2088; wyzez de walle wyn wezed to hem oft 1403, and braydez out de bryzt bronde 1901.

Superlativ: whyle de wlonkest wedes he warp on hymseluen 2025. $\mathrm{Zu}$ "as" vgl. unten!

Am schlusse dieses abschnittes sei darauf hingewiesen, dafs pron. demonstr. und best. art. sich noch so nahe stehen, daLs mehrfach in gleichen oder ganz parallelen fällen jetzt das eine und dann das andre gebraucht wird: Jat fayr lady 1208, de gay lady 1248 ("סat" nicht etwa durch kontext bedingt); 1263 gegen 1492, 1977.

B) Der bestimmte artikel fehlt. Er wird nicht gesetzt 1. im vokativ, 2 . in der verbindung adj. + eigenname, 3. beim prädikativen subst. mit nachfolgendem superlativ.

1. Beispiele für den vokativ sind: Gode sir! 811 , ähnlich 2118 ; sir swete, $2237 ; 1218,2338$. Man vergleiche damit den auffälligen gebrauch des artikels bei Shakespeare in stellen wie diesen: The last of all the Romans, fare thee well! The empress of my soul. 
2. Adj. + eigenname. Während sich in dieser verbindung der best. art. im AE. fast immer, und im Frühmittelengl. noch sehr häufig findet, wird er in späterer zeit immer seltener und fehlt im S.G. gänzlich. Riche Romulus 8, gode Gawan 109, 2214, aðel Arthure 904, dere Vter 2465; saynt gilyan 774, bi saynt Gile 1644, be sayn Ion 1788, und natürlich ohne art. 1022: đe ioye of sayn Ionez day. Bei Chaucer ist das artikellose adj. beim eigennamen das häufigere, und in Spensers $F$. $Q u$. ist mir kein fall mit art. vorgekommen. Er sagt: Proud Duessa, Una faire...

3. Die belege aus dem $S$. G. für ein prädikatives subst. mit folgendem superlativ sind: ঠaz I were burde bryztest 1283 , for he watz beste baleful and bor alder grattest 1441, und sogar: here is wayd fayrest Jat I sez Jis seuen zere 1381-82, ze ar knyzt comlokest kyd of your elde 1520. Damit stimmen die fälle überein, die Bülbring in seinen wertvollen ergänzungen zu Einenkels arbeit bietet: ðe Jridde comed efter and is wurst fikelare, uerst louerd he was in Engelond (frühmittelenglische beispiele). Die dort schon gemachte bemerkung, dafs in diesen fällen der art. nur fehlen kann, bleibt auch für unser gedicht zurecht bestehen; denn neben 754 ohne stehen 938, 1915, 2196 mit art.: and sayde he watz de welcomest wyze of Je worlde 938, hit watz de myriest mute Jat euer men herde 1915, hit it Je corsedest kyrk dat euer I com inne 2196. Nicht ganz sicher ist wegen ihrer elliptischen ausdruckweise die stelle $355 \mathrm{zu}$ bestimmen, die aber wohl prädikativ, als parallele zu 754, aufzufassen sein dürfte: $I$ am Je wakkest, I wot, and of wyt feblest, and lest luv of my lif, quo laytes ðe soðe. Man gestatte mir hier anzuschliefsen, dals bei "most" der best. art. fehlt (quo walt Jer most renoun 231, hom ... Jat most myrðe myzt meue ðat crystenmas whyle 985) und der best. art. in einem einzigen, wohl metrisch bedingten falle auch nicht steht, in dem man ihn nach dem früher gesagten erwarten könnte: Kyng hyzest mon of wylle 57 (kurze reimzeile).

Artikellose superlative bei einem subst., das aber durchaus nicht prädikativ zu sein braucht, finden sich bei Sp. häufig: Jat hardest heart would bleede VIII, 36, IX, (stinges) Jat sharpest steele exceed XI, 11, IX; ähnlich IV, 4, V. Auch in der spätern dichtung bleibt beim supl. der best. art. häufig aus, 
z. b. Paradise lost IX, 431-32: Mindless the while her self, though fairest unsupported flow'r; thee, serpent, subtlest beast of all the field I knew IX, 560; (knights) couch their spears till thickest legions close, II, 536-37; to perplex and dash maturest countels II, 114-15.

Neben den oben aufgestellten und nun belegten drei fällen, in denen der best. art. fehlt, ist bisher ein vierter unerwähnt geblieben, der aber auch besonderer hervorhebung und erläuterung kaum zu bedürfen scheint. Natürlich fehlt der art. da, wo es sich weder um hinweisung noch aussonderung handelt, vielmehr nur um unbestimmte, allgemeine angaben: Good ber and bryzt wyn 129, fayre fannand fax 181, clere lyzt 1649 ... Anstatt nach dem ersten abschnitte über stehen und dem zweiten über fehlen des bestimmten artikels nun zum dritten über stehen oder fehlen überzugehen, halte ich es für geraten, mich der behandlung des unbestimmten artikels zuzuwenden. Sonst nicht zu vermeidenden unsicherheiten mit bezug auf die verbindung präposition + adj. + subst. hoffe ich so $\mathrm{zu}$ entgehen und ermüdende wiederholung beim abschnitte "stellung. des artikels" unnötig zu machen.

b) Der unbestimmte artikel.

a) Er steht. Prüft man unter berücksichtigung der oben angenommenen unterscheidung nach zählendem, einführendem und allgemeinem unbestimmten artikel das im $S$. G. gebotene material durch und beachtet dabei den subjektiven, objektiven und prädikativen gebrauch des betreffenden substantivs, so kommt man zu dem interessanten ergebnis, dafs beim subjektiv gebrauchten subst. mit adj. der einführende, beim objektiven der zählende, und beim prädikativen der allgemeine unbest. art. entschieden überwiegt. Der dritte fall mufs bis abschnitt y) zurückgestellt werden, da sich für ihn in bezug auf setzen oder fortlassen des artikels keine regel auffinden läfst, ob er gleich geschichtlich verständlich ist. Von dem subjektiv und objektiv gebrauchten subst. aber gilt, dafs vor ihnen der art. erscheint, wenn sie nicht ganz unbestimmt und allgemein als gattungshegriffe hingestellt werden sollen. Jer hales in at de halle dor an aghlich mayster 136, a strayt cote ful strezt, Jat stek on his sides, a mere mantile ... 152-53, (i grene hors gret and Jikke 175; a much berd 182; ähnliche 
stellen: 194, 209, 807-8, 878, 2172. To bide a blysful blusch of de bryzt sunne 520, to dryze a delful dynt 560, ho razt hym a riche rynk of red golde werkez 1817. Weitere entsprechende belege finden sich: $268-9,788,1183,1195,2369$, von denen der lokale und temporale akkusativ 788 bezw. 1195 hervorgehoben seien: And eft a ful huge hezt hit (walle) haled vpon lofte; te lede lay lurked a ful longe quyle.

Als zweites akkusativobjekt begegnet " $a$ " in den versen $29,843-4,964$; z. b. 964 : A mensk lady on molde mon may hir calle. Besondere erwähnung verdient die stelle 679: $A$ lowande leder of ledez in londe hym wel semez. Mit dem adj. im komparativ merke man die verse: a better barbican dat burne blusched vpon neuer 793, dat a comlyker knyzt neuer Kryst made hem Jozt 869-70. (Vgl. aber unter $\beta$ ) bei “neuer"!) Während sich nach Einenkels angabe bei $C h$. fast überall " $a$, an" findet, unterdrückt noch Sp. nicht selten den art.: Mortall wight, living creature, valiant knight, carefull nourse, greedy gulfe, scaly tayle. Verschiedene behandlung im prädikat und beim objekt zeigt die stelle X, 10, I: Straunge thing it is an errant knight to see here in this place.

ß) Der unbest. art. fehlt. Ein gutes beispiel ist 2354: Trwe mon trwe restore. Wiewohl in dieser kurzen reimzeile metrisch ein voraufgehendes " $a$ " nicht nur zulässig, sondern sogar zu erwarten wäre, fehlt es doch: Es handelt sich eben nicht um einen einzelnen vertreter einer gattung, der zählend oder einführend durch den unbestimmten art. abgesondert wird, sondern ganz allgemein um irgend einen, um jeden redlichen mann. Aehnliche fälle sind: 64, 47.

Bemerkenswert ist die stelle $968-69$ mit dem komp., bei dem man den art. erwarten sollte: More lyliker-wys on to lyk watz dat scho hade on lode. Infolge der allgemeinheit der aussage fehlt der unbest. art. in den mit "as" eingeleiteten vergleichen: Al de gayne đou me gef, as god mon schulde 2349, ähnlich 2241, 1465; ebenso auch beim supl.: As tulk of tale most trwe and gentylest knyzt of lote 638-39. Es befremdet, daIs nach Einenkel sich bei Ch. nur der eine fall IV, 57 (as seke man hath of swete and bitternesse) findet, sonst aber der art. gesetzt wird. Dies ist um so erstaunlicher, als wir bei $\mathrm{Sp}$. noch oft genug genaue entsprechungen der stellen im S. G. haben, z. b.: As chauffed bore his bristles doth upreare 
XI, 15, VI, so far as eughen bow a shatt may send XI, 19, II, so XI, 19, V u. a. XII, 22, I fehlt der art. sogar beim supl.: As freshest flowre in may. Es sei hier angefügt, dals nach "as" im S. G. einmal der best. art. steht: As de bryzt sunne 1819, wobei darauf aufmerksam zu machen ist, dals "bryzt sunne" immer mit dem art. vorkommt; aus $\mathrm{Ch}$. biete ich zum vergleiche: as sonne bryzhte F.170, aber ebenfals mit art. F. 53: the sonne shene.

Für das fehlen des unbestimmten artikels nach "neuer", wie es sich bei $\mathrm{Ch}$. und vielen andern schon seit der Sachsenchronik beobachten läIst, bietet $\operatorname{der} S$. $G$. nur einen beleg mit attributivem adj.: Watz neuer freke fayrer fonge bitwene two so dyngne dame 1315-16. Wie früher die fälle 793 und 869 gezeigt haben, steht " $a$ " beim komp., der als objekt dem mit "neuer" verbundenen verb voraufgeht.

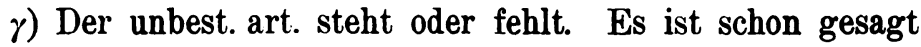
worden, dals hier in erster linie ein prädikatives subst. in begleitung eines adjektivs in betracht kommt und keine regel vorhanden ist. Wir haben es einfach mit dem beim subjektiv und objektiv gebrauchten subst. schon früh auftretenden, beim prädikativen subst. aber nur langsam vordringenden art. zu tun, der hier wohl um so mehr widerstand fand, als das prädikative adj. meist artikellos verwendet wird. Ich erinnre an die ausführung über bestimmten art. beim prädikativen subst. mit supl. Die belege mit unbestimmtem art. sind die folgenden: Hit were a pure ioye 1247, Jaze he be a sturn knape 2136, ze ar a sleper vn-slyze 1209, for he is a mon medles 2106, hit were a fole fele-folde 1545, hit were a wynne huge to luf hom wel ... 2420-21. An den folgenden stellen fehlt im prädikat der art.: Vchone .. dat watz wyze stronge 1028, I am wyze vn-uondy 1244, he watz beste baleful 1441, as I am trwe segge 1673; watz he neuer in Jis worlde wyze half so blyðe 2321.

Zahlreiche beispiele gehören einem falle an, in dem der art. stehen oder fehlen kann, nämlich der verbindung präposition + subst. mit attributivem adj. Nur die bezeichnendsten belege sollen jetzt angeführt werden, da im metrischen teile auf manches zurückzukommen sein wird, und, wie oben angedeutet, scheint es geraten, hier vom fehlen des artikels "schlechthin", nicht mehr "des bestimmten" oder "des unbe-

Anglia. N. F. $\mathbf{X X}$. 
stimmten" zu sprechen; denn trotz einiger klarer fälle ist es häufig nur möglich zu sagen, dafs der art., nicht aber, ob nun ein " $\partial "$ " oder " $a$ " fehlt.

Präposition + best. art. Wie in unabhängiger stellung steht nach präpositionen der best. art. hinweisenden charakters, wenn das subst. von einem zu- oder nachsatz begleitet ist: To Je hez lorde of Jis hous 812, in Je more half of his schelde 649 , at de fyrst queðe of ðe quest 1150; and derely hym Jonkkez of Je uynne worschip dat he hym wayned hade 103132, wyð ðe best gemmes Jat myzt be preued of prys . . 78-79.

Ebenso tritt nach präpositionen der best. art. auf, wo, auch ohne solche beifügung, hinweis auf bekanntes oder zu bekanntem in naher beziehung stehendes vorliegt: Đurz de sale riche 243 , driuande to de heze dece 222 , ähnlich 75,108 ; syn je be lorde of de zonder londe 2440; 13, 1157, 1170, 2310. Parallelfälle mit vollem demonstr. pron. sind: Of бo roz wonez 2198, at do ladyez wlonk 1977. Hinweis durch beziehung auf bekanntes: Herttez ... wið ठe hyze hedes 1154, ठat wyl I welde ... not for de wynne golde ... ne for Je wlonk werkkez (natürlich des gürtels) 2430-32. 2310. 601, 516.

Wie in freier stellung ist nach präpositionen in vielen fällen abnahme der hinweisenden kraft und wachsende ausdehnung der absoluten, nur aussondernden gebrauchsweise des bestimmten artikels zu beobachten: To $\partial e$ fale erðe 728, fast so 818 , of de softe somer 510 , of de hyze tyde 932 , to de derk nyzt $1177=1887$, vpon de gray morne 1024; in de depe sladez 1159 (keine rückbeziehung oder wirklicher hinweis), ähnlich 772, 723. 118. In đe best lawe 790 .

Der unbest. art. wird auch nach präpositionen zählend, einführend und absolut verwendet, in dem früher näher bestimmten sinne. Wið a runisch rout đe raynez he tornez 457, ähnlich 1480, 1818.

Bi a littel dich 1709, ähnlich 1710, 1712 ; bitwene a flosche in Jat fryd and a foo cragge 1430, ferner 1464, 1614, 1736 ...

Wyð a wrast noyce 1423 , fast $=1706,1905$; in a siker wyse 2048, ähnlich 2014; (ze) oghe to a zonke Jynk zern to schewe 1526.

Der art. fehlt nach präpositionen. Das gilt zunächst natürlich da, wo man ihn auch sonst nicht erwarten würde, also im unbestimmten plural: He wid his haðeles on hyze 
horsses weren 1138, wið ful stor wordez $1291 \ldots$ Doch ist das fehlen des artikels, des bestimmten wie des unbestimmten, für sing. wie pl., häufiger und regelloser, oder, geschichtlich gesprochen, das alte hält dem eindringenden neuen noch besser stand, was folgende beispiele erläutern mögen: (ðe rayn) fallez vpon fayre flat 507 gegen er hit falle myzt to de fale erde 728; wid goud wylle $1500=2430$ gegen: wid a goud wylle $1861=1969$. In ähnlicher weise 1423 gegen 1724 . Nach ähnlichkeit vieler stellen ohne und einiger mit präp. dürfte man den art. erwarten z. b. in folgenden fällen: Wið heze helme on his hede, his launce in his honde 2197, ferner 493-94, to hunt ... at hyndez barayne 1320, ferner 1207. Wyd sturne schere 334 gegen : wið a wale chere 1759. Beachtenswert sind von den zahlreichen fällen mit fehlendem art. noch: 581,1165 , 1179, 1181, 1748, 1750, 1873, 2475.

Noch bei Spenser und Milton fehlt, oft gewifs infolge des metrums, der art., sogar beim superlativ: At last faire Hesperus in highest skie had spent his lampe F. Qu. I, 2, VII; and his dark suggestions hide from sharpest sight Par. Lost. IX, 90-91.

d) Gradpartikeln und pronomina beim attributiven adj. in hinblick auf den artikel. Ein abschnitt, der sowohl unter die überschrift "der art. steht oder fehlt" gehört, als unter die andre "stellung des artikels" ist die erörterung der frage, ob und wohin er gesetzt wird, wenn einen grad bezeichnende partikeln oder adjektivische pronomina wie such, many ... vor das adj. treten. Aus dem S.G. liegt folgendes material zur beurteilung vor: So hardy a here 59 , wid so pouer a mon 1538; scho made hym so gret chere 1259; of face so fere 103, in wod so wlonk 515 ; lote so myy 1086; non so hyze hawtesse 2454. Die belege sind also spärlich, und für Jus, as, hou', to, ouer, what fehlen sie ganz. Von "as" und "how" gibt Einenkel für $\mathrm{Ch}$. an, dafs sie sich fast immer mit art. finden. Für Sp. füge ich hinzu, dafs zumal bei "so" der gebrauch des artikels schwankt: So weak an enimy VIII, 45, VIII, so fowle deformed wight VIII, 49, II, so great a weight XI, 18, VI, so great load XI, $54, \mathrm{IV}$, so rash a doome IX, 38 , II, so cruell cace XI, 26, VIII ; mit art. z. b. noch IX, 13, IX, XI, 55, IX, ohne art. VIII, 7, VII, XI, 16, VI, XI, 17, VI und XI, 17, VIII. Nach besprechung der gradpartikeln kommen wir zu "sıch, 
many und all" und finden zunächst für unsern text: Such a glauerande glam 1426, of such a sellyly soiorne 1962, ferner 92, 1166. Ohne art. kommt such vor 46 , einer genauen parallele zu 1426: Such glaumande gle. Mony. In mony a bonk vnbene 710, wið mony a blyðe lazter 1217. Aber: Mony aðel freke 241, mony wylsum way 689, mony syker knyzt 2493, ähnlich 115; of mony borelych bole 766, wyð mony luflych loupe 792, ähnlich 1601, 1707; wyð mony baner ful bryzt 117. Selbstverständlich auch kein art. beim unbestimmten plural: mony bellez ful bryzt 195. Die drei belege mit dem nicht häufigen "fele" seien hier angefügt: on fele wyse 1653, so felle flonez 1566, fele Jryuande Jonkkez 1980. Alle. Alle de godlych gere 584, wid alle ठe manerly merðe 1656; alle ठe clamberande clyffes 1722, alle de burnez so bolde 1574 .

Es zeigt sich also, dafs im $S$. G. bei "alle" der art. immer, bei "such" in fast der hälfte und bei "mony" in einem fünftel der vorhandenen fälle gebraucht wird. Bei $\mathrm{Ch}$. fehlt nach many, such und which der art. gelegentlich, bei ilk und each immer. Die weitere bemerkung Einenkels, dafs im Ae. nach âlc ganz selten der art. stehe, veranlalst mich, aus dem S.G. anzuführen: vche a cace 1266, vche a knyzt 1491. Das über gelegentlichen artikelhaften gebrauch von $\delta a t$ und $\delta i s$ besonders bei on, oðer und, ich ergänze $i l k$, gesagte bestätigt sich für unsern text: 1256, 1615, 2312, 2369, 1492. Ganz seltenes Chaucersches "all" mit unbestimmtem art. fehlt, wie man gesehen. Half, whole, boð mit nachstehendem art. desgleichen; doch sei aufmerksam gemacht auf 185: half his armes.

$\mathrm{Zu}$ dem abschnitt "stellung des artikels" gehören endlich die früher schon aufgeführten fälle von on + supl. : on $\delta e$ fautlest freke, die sich als parallelbildungen zu seltenen ae. belegen wie "ठrêo đâ betstan ele" erklären. Am schlusse dieses abschnittes sei auf die auffällige stellung des zahlworts zwischen adj. und subst. an folgenden zwei beispielen aufmerksam gemacht: bare dre dayez 1066, dagegen: two bare myntes 2352 und, ein merkwürdiger singular, dre bare mote 1141 und two so dyngne dame 1316. Das zweite vorhandene beispiel begegnet 1946: Jro suche Jre cosses. 


\section{$\S 38$. 4. Das attributive adj. in der zusammensetzung.}

Neben dem bisher behandelten und als selbständig empfundenen attributiven adj. haben wir es in einer reihe von fällen in unselbständiger verwendung, indem es, besonders infolge von häufigkeit im gebrauche und abschleifung und erweiterung des begriffsinhaltes, mit seinem subst. zu einem einheitlichen, oft neuen begriffe zusammenwächst. Da dieser vorgang manchmal noch nicht abgeschlossen ist, vermag man nicht immer mit sicherheit zu sagen, ob eine neue zusammensetzung vorliegt oder nicht. Ich beginne deshalb mit den sichern fällen und der verbindung adj. + subst.

Crystenmasse 502, cryst-masse gomnes 683; halyday 805, halidayes 1049, Holy-Hede 700; hendelayk 1228 (höfisches wesen, ritterlichkeit), die alte zusammensetzung lemman 1782; mydmorn $1073=1254$; trulofez 612 (truelove knots), trweluf craftes 1527. Weniger sicher sind folgende belege: rounde table 39, 313,538 ..., graunt mercy 838 ..., bone hostel 776 ; bele chere 1034; heze auter 593. Es stützt die annahme der zusammensetzung, dafs in allen fällen das adj. alliteriert und sich $\mathbf{7 7 6}$ und 1034 und 838 anders nicht vorkommende adjektiva finden. Wahrscheinlich hat man auch in god-day $1029=1290$, god day 2073, goud moroun 1213 eine zusammensetzung zu erblicken; überall stabt "god", 60 und 284 wird "nwe zer" kaum als ein wort empfunden sein, gewils aber 1968: on nwzerez morn.

Nur in vier beispielen ist die verbindung subst. + adj. vertreten; auf dem subst. als dem wichtigern, verglichenen teile liegt hier der hauptton. Beuer hwed 845, braymwod 1461, chalk whyt 798, mylk quyte 858.

\section{Das appositive adjektiv.}

$\S 39$. 1. Art und zahl der appositiv gebrauchten adjektiva.

Vom attributiven unterscheidet sich das appositive adj. nur dadurch, dafs es sich nicht unmittelbar an sein subst. anlehnt, sondern ihm oder dem das subst. vertretenden pron. der regel nach als zusatzerklärung nachträglich beigegeben wird und ihm so freier gegenübersteht. Auch zum prädika- 
tiven adj. liegen beziehungen vor, und zwar so enge, dafs sich zuweilen nicht entscheiden läfst, ob es sich um apposition oder um prädikat mit ellipse des verbs handelt.

Im $S$. G. kommen adjektiva in appositiver verwendung nur $49 \mathrm{mal}$ vor, also bei unsern 730 fällen von attribuierung fast im verhältnis von $1: 15$. Leider lassen die berechnungen Scheinerts für Bêowulf keinen vergleich zu, zunächst weil er nur vers $1-1250$ berücksichtigt, besonders aber deshalb, weil er auch da von appositivem oder prädikativem adj. spricht, wo es sich wirklich um ein derartiges subst. mit attributivem adj. handelt. Die für den $S$. $G$. angegebenen 49 appositiv gebrauchten adjektiva verteilen sich nach sprachlicher herkunft und bildungsart so: altengl. adjektiva 32, altfrz. 8, altn. 3, andre germ. 1, abgeleitete 4 und zusammengesetzte 1. Die sechs adjektivgruppen sind am, aufbau dieser summe so beteiligt: I. sinnenfällige eigenschaften 7, II. wesenseigenschaften 16 , III. zustandsangaben 10 , IV. verhältnisbestimmungen 3 , V. ästhetische gefühle 10 , VI. sonstiger gefïhlsanteil 3. Untersucht man wie bei der attribuierung auch hier das verhältnis der fälle, in denen sich das appositive adj. auf ein lebendes objekt bezieht, zur gesamten apposition, so findet man als ergebnis $38: 49$, also fast $7: 9$, mithin gegen die attribuierung mit $1: 4$ eine dreimal häufigere beziehung auf lebewesen. Die erklärung dieser erscheinung ist wohl diese, daโs objekte mit reichem und ausgeprägtem vorstellungsgehalt eines attributes weniger bedürfen, eine oft nicht erforderliche zusatzerklärung aber eher vertragen als andre; beides trifft für lebewesen $\mathrm{zu}$.

\section{$\S$ 40. 2. Stellung des appositiven adjektivs.}

Die regelmäfsige stellung ist, wie gesagt, die hinter dem beziehungswort und werde an folgenden beispielen erläutert: Gavoayn ðe gode 1110, Gawayn ðe blyðe 1213 ... and (he hade) an $a x$ in his oðer (honde), a hoge and vn-mete 208; and alle his fetures (were) folzande, in forme dat he hade, ful clene 145-46. Bot if ze haf a lemman, a leuer, Jat yow lykez better 1782, de stif mon hym bifore stod vpon hyzt, herre den ani in de hous by de hede and more 332-33. (Je tulk) watz tried for his tricherie, Je trewest an erde 4, hit watz de ladi, 
loflyest to be-holde, Jat . . 1187. Der fall von voraufgehender apposition ist selten, er werde erläutert an dem beispiele: Jen, brayn-wod for bate, on burnez he rasez 1461. Die übrigen und ähnlichen stellen sind: 103, 632, 693, 714. Eine doppelte apposition kommt in fünf fällen vor: $302,1881,208,717,1524$.

§ 41. 3. Gebrauch des artikels beim appositiven adj.

In 29 fällen gegen 20 fehlt der art., und von diesen 20 weisen 16 den best. art. mit deutlich hinweisendem charakter, ein fall das volle demonstrativum "ठat" auf (đise cacheres $\delta a t$ couðe 1139 und dreimal kommt der unbest. art. (209, 948, 1782 ) vor. Der komparativ begegnet fünfmal, darunter einmal mit unbestimmtem (1782) und der supl. dreimal, mit best. art. 4 und 767, artikellos 1187. Das appositive adj. ohne art. findet sich mehrfach durch gradpartikel oder adverb gesteigert, so z. b. as ze haf Jryzt me here, Jro suche dre cosses, so gode 1946-47; ähnlich 1618, 2104, 717. Mit adverb: and alle his fetures folzande ... ful clene 145-46, Jat (mantyle) watz furred ful fyne wid fellez, wel pured 1737.

Anhangsweise seien die wenigen fälle besprochen, in denen ein subst. mit attributivem adj. in apposition steht. Gawayn Je god mon 1179, Brutus, de bolde burne 2524; aber 1729: and ze he lad hem, bi-laggid mon (reniarde), Je lorde and his meyny, und besonders 57: Kyng hyzest mon of wylle.

Einenkel gibt an, dafs auch bei einem vom attribut begleiteten appositiven subst. der art. seiten sei.

\section{Das prïdikative adjektiv.}

$\S 42$. 1. Art und zahl der prädikativ verwendeten adjektiva und eigentümliches im gebrauche.

Wie vom attributiven zum appositiven adj., so liegt vom appositiven zum prädikativen ein weiterer fortschritt vor in der schärfern begrifflichen absonderung und der verselbständigung des adjektivs gegenüber seinem beziehungswort. Auf die übergänge zwischen apposition und elliptischem prädikat nnd daraus zuweilen entstehende unsicherheit für die zählung ist schon hingewiesen; jetzt ist noch hinzuzunehmen, dafs in einigen wenigen fällen zweifelhaft bleiben muls, ob man es 
mit einem bei einem verb stehenden adj. oder adv. zu tun hat. Die gesamtzahl der fälle mit prädikativem adj. zerfällt nämlich in zwei abteilungen: 1. das beim verbum substantivum, 2. das bei andern verben stehende prädikative adj. Der häufigkeit ihrer verwendung nach ergeben die beiden gruppen zusammen die zahl $255=193+62$. Das bedeutet im vergleich mit den 730 fällen von attribuierung beinahe ein verhältnis $1: 3$ und verglichen mit der apposition (255:49 $=5: 1$ ). Es bleibt mithin für den $S$. $G$. die bei Scheinert gemachte behauptung bestehen, dafs die attribuierung im Ags. die vorherrschende gebrauchsart sei, nicht aber läfst sich mehr sagen, dals die prädizierende gänzlich zurücktrete (übrigens bestätigen Scheinerts eigene berechnungen seine aussage nicht). Die angegebenen 255 stellen mit prädikativem adj. verteilen sich auf unsre bekannten sechs gruppen so: I. 34 , II. 64 , III. 76 , IV. 27 , V. 19 , VI. $35=255$. Wird auch hier die frage erwogen nach der sprachlichen herkunft und bildungsweise, so zeigt sich, dafs von belegen mit prädikativem adj. auf das Altengl. 167, auf das Altfr. 36, aufs Altn. 18, auf andre germanische sprachen 2, auf ableitungen 23 und auf zusammensetzungen endlich 9 entfallen.

Das verhältnis der fälle mit einem prätikativen, sich auf lebewesen beziehenden adj. zur gesamten prädizierung stellt sich auf $157: 255$, also fast das von $3: 5$ und somit hinter dem ergebnis für die apposition, $7: 9$, beinahe um ein fünftel zurückstehend. Ein doppeltes prädikat findet sich im $S$. $G$. 10 mal, sodals sich also zwar nicht mehr mit der Bêowulfstudie sagen lälst, dals prädikative adjektiva nie in gruppen auftreten, aber die seltenheit des falles doch deutlich zu tage tritt. Scheinert weist richtig darauf hin, dals die dinge bei Chaucer nicht so lägen und ich füge hinzu: auch nicht bei Spenser. Folgende beispiele mögen dies erläutern: (th'only good) is to be wise and ware of like agein VIII, 44, II, ähnlich VIII, 7, VI; streight and narrow was the way X, 5, IX, all were his earthly eien both blunt and bad X, 47, III.

Während es unnütz scheint, den ganz bekannten fall der prädizierung durch ein adj. und das verbum "to be" an beispielen zu erläutern, erheischt unsre zweite gruppe, adj. bei begriffsverb, aufmerksame prüfung des vorhandenen materials, das nach verbgruppen geordnet vorgeführt werden soll. Fol- 
gende überlegung scheint geeignet, an die stelle empirisch zusammengetragener wörterlisten zusammenhängende gruppen von verben zu setzen, die anstatt eines adverbs ein adj. zu sich nehmen: die dem verbum substantivum nächststehenden und zwischen ihm und den eigentlichen begriffsverben vermittelnden wörter sind bezeichnungen für zustände und arten des seins. Das sein und seine zustände gegeben, folgt eine zweite verbgruppe mit aussagen darüber, wie es uns in der wahrnehmung affiziert, dann eine weitere, wie wir uns ihm gegenüber denkend und urteilend verhalten, und schliefslich eine letzte gruppe des inhalts, wie wir im handeln auf die objekte einzuwirken suchen. Natürlich können diese vier gruppen in einem einzelwerk nur stückweise vertreten sein, und gleich der ersten gehören nur vier sichere fälle an: ðe sauer to worðe 1202, (heruest) warnez hym for de wynter to wax ful rype 522, to grow grene 235, and me als fayn to falle feye as ... 1067. Nur zögernd kann man hierher stellen 2392: (бou) hatz de penaunce apert of de poynt of myn egge, und noch unsicherer sind die stellen: der-fore of face so fere, he stiztlez stif in stalle, ful zep in бat $n w$ zere 103-105 und: al stonstil seten 242. Man könnte geneigt sein, in "stif, zep, stonstil" adjectiva zu sehen auf grund ae. fälle wie: uplang âstôd, oððät îdel stôd hûsa sêlest und Chaucerscher beispiele nach art des folgenden: his stede, which that shoon as sonne bryghte stant in the courte, stille as any stoon Gr. F. 170-71. Aus dem S. G. selbst aber sprechen folgende stellen sehr dagegen: how Jat dozty dredles deruely der stondez 2334, (ठay) seten soberly 940, (ho) set hir ful softly 1193. Die zweite gruppe von verben, bei denen adjektiva stehen, enthält auf sinneswahrnehmung bezügliche begriffe; sie ist in unserm falle wieder nicht grofs: he loked as layt so lyzt 199, clad wyd a clene cloðe, ðat cler quyt schewed 885, and ay de lady let lyk = sah vergnügt aus 1281, he sez hir so glorious, and gayly atyred, so fautles of hir fetures ... 1761. Vielleicht ist dieser gruppe zuzuzählen 269: a schelde and a scharp spere, schinande bryzt.

Die verba des denkens und sagens der dritten reihe sind zahlreicher vertreten und so im einzelnen belegt: and yow god Jozt 1245, lef hit me Jynkes 1111, ähnlich, 49, 348, 819, $909,1241,1620,1793,2109,2163,2170$ und 2167 und 1578, die vor den andern hervorgehoben zll werden verdienen: $\delta e$ 


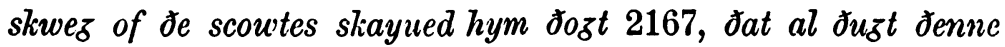
ful loðe be more wyd his tuschez torne 1578-79. As frekez Jat semed fayn 840, to ryche for hit (rynk) semez 1827. If any so hardy in ois hous holdez hym-seluen 285, and oy burz and $\delta y$ burnes best ar holden, stifest vnder stel-gere on stedes to ryde, ðe wyztest and ðe worठyest of ðe worldes kynde 259-61. Die übrigen ähnlichen stellen findet man: 1274, 2097, 2270, 2390. Hit is soð knawen 348, Gawan watz for gode knawen 633. And faydful I fynde ठe 1679, fyrst he watz funden fautlez in his fyue uyttez 640 , aulserdem 1264 . ze demen me to dille to ... 1529. Jer-fore com, oder recreaunt be calde Je be-houeus 456. I wowche hit saf fynly 1391 (vgl. noch bei Sp. X, 64, VIII: that word shal I, said he, avouchen good). Während "vouchsafe" fortlebt, ist das ebenfalls dem Afr. entlehnte "quit-clayme", clamer quite, ausgestorben, aber S.G. 293: I quit-clayme hit for euer.

Zur vierten aufgestellten gruppe gehören verba des handelns wie die folgenden: weldez non so hyze hawtesse, Jat ho ne con make ful tame 2454-55, de lorde hit tayt makez 988, ঠay made hem blyðe 1398, make we mery 1601: wenn auch das pronominalobjekt, hit oder us, fehlt, so ist doch die auffassung wohl die gleiche; ähnlich 1953; 1313, 2468 ohne jedes pron.: He sette hym so clene as . . $1883=$ machte ihn so rein. And syden rendez him (bor) al roghe 1608; and zet hem halchez al hole đe haluez to-geder 1613, ähnlich 1346: and heuen hit vp al hole. (Je wynde) drof vche dale ful of dryftes ful grete 2005. Das unter 2. schon aufgeführte "schewe" begegnet hier aufs neue, nun aber nicht mehr in der bedeutung aussehen, sondern in der von schauen machen, zeigen: (he) schewed Jat schyre al bare 2256, ðe nirt in Je nek he naked hem schewed 2498; über alone vgl. man weiter unten!

Zum prädikativen adj. bei verben sei abschliefsend auf folgende besonderheiten aufmerksam gemacht: Die gruppen 1 und 2 zeichnen sich 3 und 4 gegenüber dadurch aus, dals hier fast ausschliefslich intransitive, und dort beinahe nur transitive verba begegnen. 3 wiederum hat das unterscheidende merkmal vor 4, dals zahlreiche, dort ganz fehlende unpersönliche ausdrücke vorkommen, während ihnen beiden gemeinsam ist der häufige gebrauch des doppelten akkusativs und auch nominativs. 
Im gegensatz zur prädizierung mit "to be", für die sich das verhältnis des engl. anteils zu dem aller übrigen auf fast $5: 3$ (121:72) stellt, ergibt eine aufstellung nach gleichen gesichtspunkten für das adj. beim verb die werte von nahezu $3: 1$ (46:16). Ein letzter vergleich, nämlich hinsichtlich der beziehung des prädikativen adjektivs auf ein lebendes oder sonstiges subjekt lehrt, dals bei der einfachen prädizierung ein verhältnis von fast $2: 1$ (128:65), bei der mit begriffsverben aber ein solches von beinahe $1: 1(30: 32)$ herauskommt.

$\mathrm{Zu}$ eigentümlichkeiten in der gebrauchsweise einzelner adjektiva übergehend, beginne ich mit den anderswo schon gemachten einschlägigen bemerkungen. Scheinert: Nur prädikativ verwendet werden angaben allgemeiner relationen, impersonalia und verbaladjektiva. Einenkel weist auf den im prädikat vor sich gegangenen funktions- und bedeutungswechsel von still, quite, fayne, holde, unwiste und ded hin. Aus der reihe der bei Wendt genannten prädikativen ausdriicke hebe ich hervor: alone, afraid of, beholden to und die bemerkung, dafs little, von besondern umständen abgesehen, sich im prädikat selten finde, da es dann absolut klein und winzig bedeute. Diesen am schnellsten zu erledigenden punkt gleich vornehmend, werde festgestellt, dafs im $S$. G. littel im prädikat nur einmal, 1848, vorkommt und dort wirklich "wenig, gering" bedeutet. Den drei aufgeführten "fayne, stille, quite" ist gemeinsam, dals sie eine entwicklung zum adverb durchgemacht haben, wobei "fayne" den weitesten weg, den vom partizip und adj. zum adv., durchlaufen hat. Während Ch. es meist schon als adv. braucht, kennt S.G. es nur als adj.: as I am ferly fayn, Jat . . 388, frekez Jat semed fayn 840, me als $f$. to falle feye, as .. 1067, he watz $f$. Jenne to Jonk 2019. Spenser hat das wort in beiden verwendungen, als adj. z. b. X, 62, V: but deeds of armes must $I$ at last be faine and ladies love to leave? XI, 37, VII: that to his force to yiclien it (air) was faine; als adv. z. b. IX, 25, III: eft looking back would faine have runne away. Bez. des adjektivs beachte man den schon am letzten aus dem $S . G$. angeführten beispiele wahrzunehmenden bedeutungsübergang von froh über freudig bereit zu bereit. Das von Ch. schon im sinne von dennoch gebrauchte "stille" kommt so noch 
nicht im S. G. vor: ze wyl be stylle 1996 und 301 der komparativ. Der wandel ist nach Einenkel vor sich gegangen von der redeweise aus: to ben, to let be stille $=$ in ruhe, immerhin, dennoch sein oder lassen. Solche zwischenstufen der entwicklung liegen noch in den Spenserbeispielen vor: whose aunswere bad me still assured bee, IX, 5, VII, still = immerzu, fortwährend XI, 13, VIII; XI, 32, IX. "quite" kommt im $S$. G. nur unselbständig in der schon angeführten zusammensetzung "quit-clayme" 293 vor. "unwiste", bei $\mathrm{Ch}$. meist adv., ist unbelegt. Wie bei Ch. haben im $S$. G. die partizipien halden, bihalden, biholde die adjektivische bedeutung verbunden, verpflichtet angenommen: I am derely to yow biholde 1842, ähnlich 1547, 1040. Der entgegengesetzte wandel von adjektivischer zu partizipialer bedeutung liegt, wie häufig bei $\mathrm{Ch}$., so bei uns für "ded" vor: nade he ben duzty and dryze, and dryztyn had served, douteles he hade ben ded, and dreped ful ofte 724, 25 : also nicht "tot", sondern "getötet"; ganz ähnlich 2264. Ich erinnre zurück an eine in der stilistik besprochene dritte verwendungsweise von "ded" bei Ch., nämlich als "tötlich": woundes dede III, 1211. Die längste entwicklung hat unser heutiges prädikatives "alone" hinter sich. Es ist hervorgegangen aus dem als adjektivischen pron. gebrauchten zahlwort one, verstärkt durch das adv. al, vermindert aber um das zugehörige persönliche pron. Das gesagte wird sogleich klar werden an den von mir gesammelten beispielen: for unc ânum twâm = uns beiden allein, (runic wand), ân sceal inbindan forstes fetre fela-meahtig god; earm biðse đe sceal âna lifgan (beide in "gnomic verses"). S. G.: we (ar) bot oure one 1230, jat (court) aðel Arthure ðe hende haldez hym one 904, let Je gome one 2118, and (he) leuez Je knyzt dere al one 2154-55, to kayre al his one 1048 (wohl aus mifsverstandener konstruktion). Leider merken ten Brink und Einenkel über alone bei $\mathrm{Ch}$. nichts an, und mir bleibt, anstatt allgemeine angaben zu machen, somit nur, auf fälle aus eigener lektüre hinzuweisen, wie z. b. Out of his dores anon he hath him dight alone ..., B. 3719-20. Beachtenswert erscheint: And as a widdow was she, and all alone, Tr. and Cr. 97. Aus Sp. hebe ich die besonders auffällige stelle VIII, 18, II heraus: in one alone left hand; auffällig erstens wegen der durch die verdoppelung des "one" bewie- 
senen begriffseinheit von "alone" und dann wegen des attributiven gebrauches. Damit halte man zusammen die stelle aus dem S.G. 748-49: de gome vpon Gryngolet glydez hem vnder, Jurz mony misy and myre, mon al hym one. Der prädikativen nahe steht die appositive verwendungsweise im verse 693: oft, leudlez alone, he lengez on nyztez. Anhangsweise sei erwähnt, dals Milton gerne anstatt "alone" das lat. "sole" verwendet: he will reign sole king, $P$. L. II. sole wonder, IX, 533.

\section{$\S$ 43. 2. Stellung des prädikativen adjektivs.}

Von der regelmäfsigen stellung subjekt, verb, prädikatives adj. verdienen nur einige fälle besondre beachtung. ठat (burnez) brozt hym to a bryzt boure, Jer beddyng watz noble 853: wir sind also der attribuierung ganz nahe. Steigernde partikel oder adv. finden sich zuweilen von ihrem adj. abgetrennt: so reniarde watz wyle 1728, (he rydes) into a forest ful dep, Jat ferly watz wylde 741. Die häufigste abweichung von der regelmälsigen stellung besteht darin, dafs das adj., anstatt dem verb zu folgen, ihm voraufgeht, wobei dann wieder die hier aber nicht wesentlichen varianten auftreten können: adj., verb, subj.; adj., subj., verb; subj., adj., verb. Die mehrzahl der fälle erklärt sich aus metrischen, ein teil aus stilistisch-rhetorischen gründen und der rest eben aus zufälligen anlässen, gelegen in der leichten möglichkeit freierer wortfügung. Als metrisch bedingt lassen sich so viele umstellungen behaupten, weil sich zeigen läfst, daIs sie des rhythmus halber vorgenommen sind und das adj. oft den hauptstab trägt. Z. b. Miry watz de mornyng, his mounture he askes 1691: dem rhythmischen bau der stabzeile gewils angemessener als: ðe mornyng watz miry, his mounture he askes. "ze, be God", quod Gawayn, "good is your speche, bot đrete is vn-ðryuande ... 1498, 99: man denke sich wie "Jrete is vn-dryuande" "your speche is good" und der vers ist hin. Die weiteren belege für die stellung A. V. S. sind: im ersten halbvers: 569, 1447, 1536, 1660, im zweiten: 251, 508, und mit übergang in den folgenden vers 301-02. Die anordnung A.S. V. findet sich z. b. in den versen 1891 (reimzeile), $2100 \mathrm{a}, 1391 \mathrm{~b}, 1827 \mathrm{~b}$. 
Als vertreter diene 1827: if ze renay my rynk, to riche for hit semez. Zahlreich sind die fälle in der anordnung S. A. V., die sich fast immer im zweiten halbvers findet: 371, 527, 774, $877,1251,1792,2036,2233,2264,2289,2466$; über einen ganzen vers sich erstreckend: 653, 1956. Beisp.: and al grayes de gres, Jat grene watz ere 527, his clannes and his cortaysye croked were neuer $653.371,774,877$ vereinigen zwei voraufgehende subjekte in einem nachfolgenden "boðe": Jat his hert and his honde schulde hardi be boðe 371. Als beispiele für stilistisch bedingte stellung, die nur schwer als solche zu behaupten ist, können wohl gelten: gret is đe gode gle, and gomen to me huge 1536, and more he is Jen any mon vpon myddelerde 2100, sinn und metrum wirken wohl zusammen 251: and rekenly hym reuerenced, for rad was he neuer.

Wir kommen zur weitern möglichkeit, dals das adj. von zwei verben eingeschlossen ist. Eine kleine anzahl von fällen, vertreten durch beispiele wie diese: (if) ze wolde not so hyzly halden be to me 1828, bot if ðe donðe had doted, oðer dronken ben oðer 1956. Das für das prädikative adj. bei "to be" gesagte gilt auch für das mit begriffsverben verbundene, doch kommt die stellung A. V. S. nicht vor. Zur veranschaulichung genügt gewifs folgendes: ze demen me to dille 1529; lef hit me Jynkes $1111 \mathrm{~b}$, as leuest him Jozt $49 \mathrm{~b}$, hym Jynk as queme hym to quelle, as quyk go hym seluen 2109; dat ryol red cloðe, Jat cler quyt schewed 1885, and $\partial y$ burz and $\partial y$ burnes best ar holden 259; as hit is soð knawen $348 \mathrm{~b}$, Jat (Gawayn) is so goud halden. Wenn anstatt eines adjektivs, wie bisher immer, zwei im prädikat stehen, so ist an sich eine ganze reihe von möglichkeiten gegeben; der $S$. $G$. weist nur folgende fälle auf: Jat (diamauntez) bođe were bryzt and broun 618, ähnlich 966, 2099, 1525, nade he ben duzty and dryze 724, ähnlich 2382, (Jat bor) breme watz and brayn-wod boðe 1580, ðenne watz Gryngolet grayðe, ठat gret watz and huge 2047. Bei andern verben begegnet nur einmal ein doppeltes prädikat: for $\delta y$ burz and $\delta y$ burnez best ar holden ... de wyztest and ðe wordyest of $\partial e$ worldes kynde 259, 261. Manchmal liegt ellipse des das subjekt mit seinem prädikativen adj. verbindenden hilfsverbums vor, und zwar in milderer form bei kurz voraufgehenden verb: and more he is den any mon vpon myddel- 
erde, and his body bigger Jen Je best foure 2100-01, ähnlich 1171, oder ausgeprägter und abgerissener, wie $\mathrm{z}$. b. Je mane of Jat mayn hors much to hit lyke 187, and me als fayn to falle feye 1067, ähnlich 847, 1826 und mit doppeltem prädikat 138, 39. Man merke besonders 1623: de lorde ful lowde with lote, and lazed myry; hier möchte Morris latede = cried einfügen.

Von vergleichen mit andern werken mufs ich diesmal leider absehen, da man, wo von stellung gesprochen wird, überall nur an das attributive, kaum an das appositive und gar nicht an das prädikative adj. zu denken scheint, ich aber unmöglich das ganze material selbst sammeln kann.

\section{$\S$ 44. 3. Der artikel beim prädikativen adj.}

Es gilt im allgemeinen durchaus die regel, dafs das prädikative adj. ohne art. steht. Besondere aufmerksamkeit erheischt indes der superlativ, für den die belegstellen aus dem S. G. zunächst einfach aufgeführt seien: dat (holyn bobbe) is grattest in grene, when greuez ar bare 207 "Jat is a worde", quod Jat wyzt, "ठat worst is of alle 1792; I am de walkkest, $I$ wot, and of wyt feblest 354 ; bot of alle dat here bult of Bretaygne kynges ay watz Arthur de hendest 25-26, ho watz Je fayrest in felle, of flesche and of lyre 943, his mensk is de most 914. and $\delta y$ burg and $\delta y$ burnes best ar holden, stifest vnder stel-gere on stedes to ryde, Je wyztest and Je wordyest of $\partial e$ worldes kynde 259-61. In den erstgenannten beispielen, 207 und 1792, haben wir es mit der alten artikellosen gebrauchsart zu tun, während 26 und 261 der art. demonstrativen, und 354, 914 und 943 der art. identifizierenden charakters sich findet. Von den beispielen 354 und $259-61$, die superlative mit und ohne art. in sich vereinigen, sei besonders das letzte wegen des passiven verbs "ar holden" hervorgehoben. Während nämlich im Frühmittelengl. beim passiv der art. noch fehlt, wird er später häufig gesetzt, wie die belege von Bülbring und Einenkel lehren (früh z. b. he wes imaked earmest alve Jinge). Als ergänzende beispiele darf ich aus meiner sammlung wohl anfügen: dôm bið sêlast, râed bið nyttost, gnomic verses 2 , mê is đät hearma mâest, holy 
rood, the first of them, that eldest was and best, F. Qu. X, 37, I, Par. Lost IX, 163: I who erst contended with gods to sit the highest, ähnlich II, 223-24; 230.

\section{$\S 45$. Einzelerscheinungen aus dem gebiete der attribuierung und prädizierung.}

Es kommt mehrfach vor, dafs eine andre wortart die funktion des adjektivs übernimmt, dafs wir es mit einem "uneigentlichen adj." zu tun haben, wie Wendt sagen würde, der diesen abschnitt mit bemerkenswerter vollständigkeit behandelt hat. Von den dort angeführten drei fällen des ersatzes durch adv., subst. oder zusammengesetzten ausdruck koinmt für den $S$. $G$. wesentlich nur zwei, in ganz geringem malse auch drei in betracht. Ich darf aber hier zurückverweisen auf den schlufs der materialsammlung und der stilistik, wo man auch das von Wendt nicht gebotene, von mir als "umschreibung des adjektivs" bezeichnete findet. Der erinnrung diene indes: đe wynne worschip 1012, an outtrage auenture 29, wela-wylle watz de way 2084, be not so gryndel 2338; fele kyn fisches 890 , speches of myerde 860, オe cercle watz more o prys 615 . Adjektivisch gebrauchte adverbien, wie NE. the then embassador .... kommen im $S$. $G$. nicht vor. $\mathrm{Zu}$ dem bei Wendt genannten füge ich aus Milton "oft" hinzu: by oft experience (Samson Agonistes). Von dem, was Kellner als "modern overcharge of the adjectival phrase" bezeichnet, ist in unserm gedicht nichts zu spüren; Milton aber sagt z. b. with this once thy glorious champion (Sams. Agon.). Man erinnre sich hier gewisser altenglischer verbindungen aus adj., art., pron. und subst., wie z. b. ymbe heora Jät mâeste bismer (Oros. 146, 28), on đâm his unscyldigan dêaðe (Beda 553, 23). Bei Spenser findet man noch ziemlich häufig ähnliches, z. b. that her sacred book X, 19, I.

Die negation des adjektivbegriffs weist im $S$. G. nichts eigentümliches auf; weder kommt die im Altengl. oft so seltsam anmutende verneinung zur bezeichnung der bejahung vor, noch finden sich, was hier zwar nur mittelbar erwähnt werden kann, die Milton ziemlich geläufigen verneinungen mit in- statt mit -un. Einem subst. mit nachstehenden supl. 
DAS ADJEK'TIV IN "BYK GAWAYN AND THE GRENE KNYZT“. 189

entspricht im Ae. vielfach der gen. des substantivs + supl., swêga mâest, gebreca hlûdast (Riddle 4) hûsa sêlest (Bêow. 285) ... Zum schlusse sei auf folgende prädikative wendung aufmerksam gemacht: bot hit ar ladyes in-noze, Jat leuer wer nowde, haf de hende in hor holde, as I de habbe here, ... Jen much of ðe garysoun oðer golde dat ठay hauen 1251-55. Wir haben hier also übertragung der persönlichen konstruktion "I haue lef" auf die eigentliche unpersönliche "me is lef". Eine verquickung beider führt $\mathrm{zu}$ dem Chaucerschen verse: al had hir lever han had a knave child.

(Schlufs folgt.)
Godesberg $\mathbf{A} /$ Rhein.
K. Schmittretz. 